\title{
From Clinical Trial to Clinical Practice: Assessing the External Validity of Endovascular Treatment in Acute Ischemic Stroke
}

Tia Chakraborty ${ }^{1}$, Curtis G Benesch ${ }^{2}$, Adil Ali ${ }^{3}$, James P Klaas ${ }^{1}$, Waleed Brinjikji ${ }^{4}$, Justin V Chandler ${ }^{2}$, Tarun Bhalla ${ }^{5}$, Harry $\mathrm{J} \mathrm{Cloft}^{4}$, Giuseppe Lanzino ${ }^{6}$, David F Kallmes ${ }^{4}$, Bogachan Sahin², Alejandro Rabinstein ${ }^{1}$ and Eugene L Scharf ${ }^{1}$

${ }^{1}$ Department of Neurology, Mayo Clinic, Rochester, Minnesota, USA

${ }^{2}$ Department of Neurology, University of Rochester Medical Center, USA

${ }^{3}$ Center for Neural Development and Disease, University of Rochester Medical Center, Rochester, USA

${ }^{4}$ Department of Radiology, Mayo Clinic, Rochester, Minnesota, USA

${ }^{5}$ Department of Neurosurgery, University of Rochester Medical Center, USA

${ }^{6}$ Department of Neurosurgery, Mayo Clinic, Rochester, Minnesota, USA

\begin{abstract}
Background: Endovascular treatment (EVT) for acute ischemic stroke is guided by clinical trials assumed to have high external validity. To our knowledge, this assumption has not been rigorously examined.

Methods: Patients from two U.S. academic comprehensive stroke centers receiving endovascular treatment for acute ischemic stroke were retrospectively reviewed from January 2012 through December 2016. Clinical characteristics, treatment times, neuroimaging findings, and functional outcomes were compared to data from the pragmatic EVT trial, MR CLEAN. External validity was assessed quantitatively and qualitatively.

Results: 236 patients presenting with anterior circulation large vessel occlusion were included. 63\% (148/236) would have met criteria for MR CLEAN; the primary reason for exclusion would have been a time from symptom onset to groin puncture greater than 6 hours. Ordinal shift analysis of 90 day modified Rankin scale (mRS) of our clinical practice did not differ from the intervention arm of MR CLEAN, $(p=0.26)$. Favorable outcome (mRS $0-2)$ occurred in $35 \%$ of our combined practice and in $33 \%$ of the trial intervention arm $(p=0.63)$. The rates of hemicraniectomy $(3 \%$ vs. $6 \% p=0.09)$ and $\mathrm{PH}-2$ type symptomatic intracerebral hemorrhage $(5.1 \%$ vs. $6.0 \% \mathrm{p}=0.55)$ did not differ between the two groups.

Conclusion: Our clinical experience was comparable to MRCLEAN, supporting the external validity of this trial to clinical practice. We treated patients who would have been excluded from this trial because of a longer time to groin puncture. Satisfactory results can be obtained with endovascular treatment, even when treating patients beyond 6 hours from symptom onset.
\end{abstract}

Keywords: Indovascular; Ischemic stroke; External validity

\section{Introduction}

The external validity of a clinical trial is an important consideration when translating the findings into clinical practice [1]. Relevant to the endovascular management of acute ischemic stroke, treatment decisions for the application of endovascular treatment (EVT) for large vessel occlusion are based upon randomized controlled trials that demonstrated improved 90-day functional outcomes for patients receiving the intervention. Two trials EXTEND-IA [2] and SWIFT PRIME [3] enrolled patients only receiving intravenous recombinant tissue plasminogen activator (IV t-PA). ESCAPE [4] only included patients who could be rapidly triaged from door to groin in less than 60 minutes and employed multi-phase computed tomographic angiography (CTA) for patient selection. The most pragmatic of the EVT trials, MR CLEAN [5] had the broadest inclusion criteria and possibly represents the closest trial generalizable to clinical practice. MR CLEAN randomized 500 patients at 16 centers over 3.3 years, (9-10 patients per center per year). However, the lack of screening logs raises the risk of sampling bias.

Furthermore, differences in healthcare delivery between the U.S. and the Netherlands may manifest as different workflow times, treatment protocols, and patient selection, limiting the generalizability to an American context. Finally, baseline demographics and clinical characteristics of routine practice have not been compared to the trial population. These possible variations raise the question of whether EVT outcomes reported in clinical trials have external validity, and whether EVT trial participants are representative of all eligible patients with large vessel occlusion. This comparison has not yet been rigorously examined to the best of our knowledge. We compared a combined 5-year experience of 196 patients undergoing EVT at two academic comprehensive stroke centers in the U.S. to the pragmatic trial, MR. CLEAN.

\section{Methods}

Standard protocols approvals, registrations, and patient consents

This study was approved by the University of Rochester Research Subjects Review Board (RSRB\#62214) and by the Mayo Foundation Institutional Review Board (IRB16-001322) as a minimal risk protocol. Results of this study were combined in a de-identified password encrypted database for final analysis and report. Patient identifiers present in existing intra-arterial therapy databases at two academic comprehensive stroke centers were collected and reviewed for all

*Corresponding author: Tia Chakraborty, Department of Neurology, Mayo Clinic, Rochester, Minnesota, USA, Tel: 507-538-1038; E-mail: Chakraborty.Tia@mayo.edu

Received October 10, 2018; Accepted December 27, 2018; Published December 29, 2018

Citation: Chakraborty T, Benesch CG, Ali A, Klaas JP, Brinjikji W, et al. (2018) From Clinical Trial to Clinical Practice: Assessing the External Validity of Endovascular Treatment in Acute Ischemic Stroke. J Neurol Disord 6: 398. doi:10.4172/23296895.1000398

Copyright: (c) 2018 Chakraborty T, et al. This is an open-access article distributed under the terms of the Creative Commons Attribution License, which permits unrestricted use, distribution, and reproduction in any medium, provided the original author and source are credited. 
patients receiving endovascular treatment (EVT) for acute ischemic stroke between January 2012 and December 2016. Patients with posterior circulation occlusions were excluded from this study. Clinical and neuroimaging data were abstracted from the medical record and results were described. Inclusion criteria for this study were: 1) age over $18,2)$ occlusion of anterior circulation large vessel involving the middle cerebral artery (MCA) and/or internal carotid artery (ICA) and, 3) mechanical thrombectomy using modern stent-retriever or aspiration devices.

\section{Assessing external validity}

To estimate the external validity of the pragmatic EVT trial MR CLEAN, we compared the published results to our combined clinical experience with respect to baseline demographics, clinical course, recanalization rate, functional outcomes, and complication rates. For comparison, we determined what proportion of the clinical practice would have met criteria for enrollment in MR CLEAN. The primary outcome was ordinal shift analysis of the 90-day modified Rankin Scale (mRS) compared to the published intervention arm of MR CLEAN, and proportional analysis of a dichotomized functional outcome of modified Rankin scale score (mRS) of 0-2 (favorable) versus mRS 3-6 (poor) between our clinical practice and the MR CLEAN intervention arm. A subgroup analysis included patients presenting in the extended time window, defined as time from symptom onset to groin puncture greater than 6 hours, and compared to the recently presented extended time window embolectomy trial, DAWN [6]. Significance was defined as $\mathrm{p}<0.05$ and no correction was made for multiple comparisons.

\section{Clinical course}

Clinical characteristics including age, gender, atrial fibrillation, diabetes and and/or prior stroke; National Institutes of Health Stroke Scale (NIHSS); and IV t-pa were recorded. Time of symptom onset or time last known well to thrombolysis was calculated. Initial head CT for each patient was graded using the Alberta Stroke Program Early CT Score (ASPECTS) score $[7,8]$. Groin puncture times were recorded, and site of vessel occlusion was determined by review of CTA source images and/or conventional angiography images obtained during thrombectomy (ES, TC). Vessel recanalization was abstracted from the procedure report and confirmed on imaging (ES, TC) using established Thrombolysis in Cerebral Infarction (TICI) criteria [9]. Procedural complications recorded included $\mathrm{PH}-2$ type symptomatic intracerebral hemorrhage and hemi-craniectomy. Symptomatic intracerebral hemorrhage was defined by SITS-MOST criteria [10] as any radiologic hemorrhagic transformation $(\mathrm{PH}-2)$ with clinical worsening corresponding to an increase of NIHSS of $\geq 4$ within 7 days of endovascular therapy. Stroke mechanism was categorized as largevessel arterioembolic, cardioembolic, cryptogenic, hypercoagulable or other. A cardioembolic mechanism was assumed if the patient had known atrial fibrillation regardless of the international normalized ratio (INR), intracardiac thrombus, or new onset atrial fibrillation detected within 3 months of the index event in the absence of greater than $50 \%$ carotid stenosis ipsilateral to the occluded vessel. Functional outcome was defined as the 90 -day mRS [11,12] completed as determined by a certified RN, or retrospectively by chart review (ES, TC).

\section{Statistical Analysis}

Categorical variables and dichotomized variables were analyzed using chi-squared analysis. Continuous variables were analyzed using a student's t-test. An ordinal regression analysis was performed to study differences in mRS between the MR CLEAN study and our combined experience. All statistical analyses were performed using JMP13.0 (www.jmp.com, Cary, NC).

\section{Results}

The baseline demographics, neuroimaging findings, clinical course and functional outcomes for 236 patients with anterior circulation large vessel occlusions are summarized in Table 1 . Overall, median age was 71 years and median NIHSS was 19 . Atrial fibrillation (34\% vs. $25.8 \%$, $\mathrm{p}<0.005)$, diabetes $(25 \%$ vs. $13 \%, \mathrm{p}<0.005)$, and prior stroke $(13 \%$ vs. $9.4 \%, \mathrm{p}=0.046$ ) were significantly more prevalent in the clinical practice group. Concurrent IV t-pa was used significantly less often in the clinical practice group ( $45 \%$ vs. $87 \%, \mathrm{p}<0.001$ ). Median ASPECTS score was 9 at both institutions. Median time from symptom onset to groin puncture was longer in the clinical practice group (292 minutes vs. 260 minutes). The proportion of patients with favorable recanalization (defined as TICI score of $2 \mathrm{~b}$ or 3 ) was significantly higher in clinical practice $(74.6$ vs. $58.7 \%, \mathrm{p}<0.001)$. As indicated in Figure 1 , the distribution of 90 -day $\mathrm{mRS}$ did not differ between our clinical practice and MR CLEAN ( $p=0.26$ ). Note that the 90 -day mRS in four patients in the clinical practice group were lost to follow up. Furthermore, the two groups had a similar proportion of favorable outcomes defined as mRS $0-2$ (35\% vs. $33 \%, p=0.63$ ). Rates of hemi-craniectomy ( $3 \%$ vs. $6 \%$ $\mathrm{p}=0.09)$, type $\mathrm{PH}-2$ symptomatic intracerebral hemorrhage $(5.1 \%$ vs.

\section{Modified Rankin Scale}

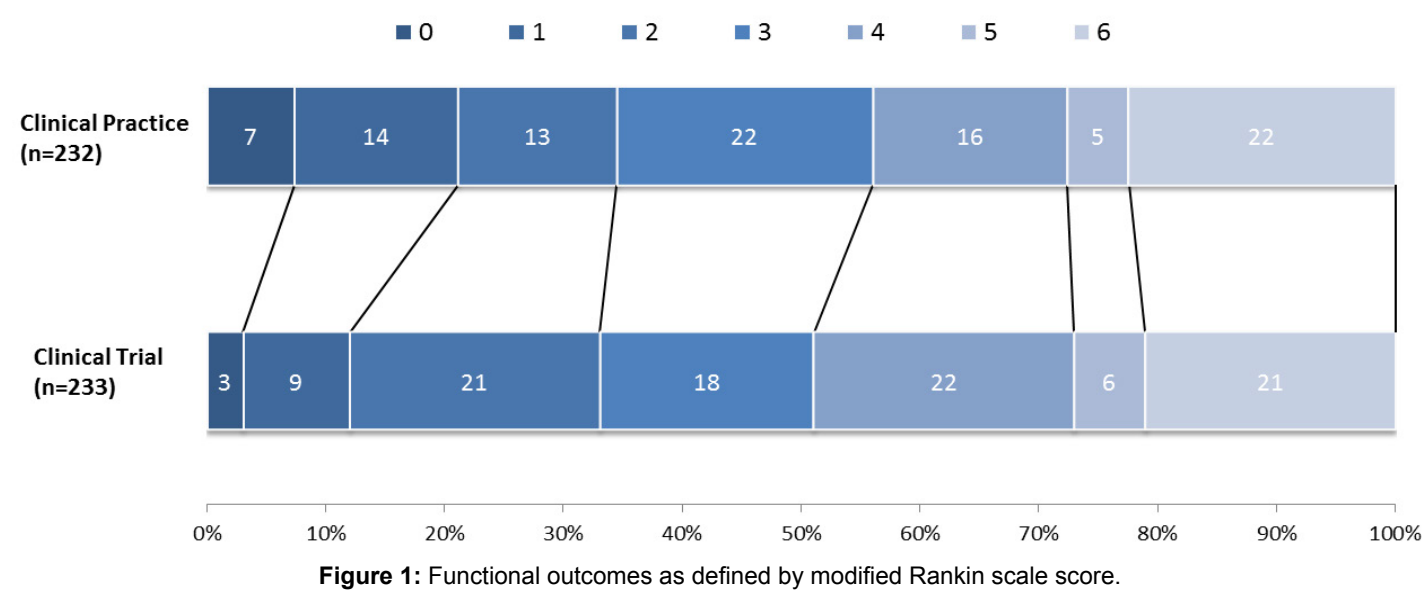




\begin{tabular}{|c|c|c|}
\hline \multirow{2}{*}{ Variables } & Clinical Practice & Mr. Clean \\
\hline & $\mathrm{N}=\mathbf{2 3 6}$ & $\mathrm{N}=\mathbf{2 3 3}$ \\
\hline Age (years), Median & 70 & 66 \\
\hline Male / Female & $113 / 123$ & $135 / 98$ \\
\hline \multicolumn{3}{|c|}{ NIHSS } \\
\hline Median (IQR) & $19(13-21)$ & $17(14-21)$ \\
\hline Range & $1-33$ & $3-30$ \\
\hline \multicolumn{3}{|c|}{ Comorbidities (\%) } \\
\hline Atrial fibrillationa ${ }^{a}$ & 34 & 26 \\
\hline Diabetes $^{a}$ & 25 & 13 \\
\hline History of stroke ${ }^{b}$ & 13 & 9 \\
\hline \multicolumn{3}{|c|}{ Mechanism (\%) } \\
\hline Cardioembolic & 57 & - \\
\hline Large vessel & 19 & - \\
\hline Cryptogenic & 23 & - \\
\hline Hypercoagulable, infectious, atypical & 1 & - \\
\hline \multicolumn{3}{|c|}{ Clinical Course } \\
\hline Treated with alteplase $(\%)$ & 45 & 87 \\
\hline Time from onset to start of alteplase (minutes), median (IQR) & $113(84-150)$ & $85(67-110)$ \\
\hline CT ASPECTS, median (IQR) & $9(8-10)$ & $9(7-10)$ \\
\hline Onset to groin puncture (minutes), median (IQR) & $292(192-441)$ & $260(210-131)$ \\
\hline General anesthesia $(\%)$ & 44 & 38 \\
\hline \multicolumn{3}{|c|}{ Location of occlusion $(n)$} \\
\hline Intracranial ICA and carotid T & 52 & 60 \\
\hline $\mathrm{M} 1$ & 162 & 154 \\
\hline M2 & 19 & 18 \\
\hline $\mathrm{A} 1$ or $\mathrm{A} 2$ & 2 & 1 \\
\hline Extracranial ICA & 15 & 75 \\
\hline \multicolumn{3}{|c|}{ TICI Score ${ }^{d}(\%)$} \\
\hline 0 & 11 & 14 \\
\hline 1 & 3 & 5 \\
\hline $2 a$ & 10 & 22 \\
\hline $2 b / 3$ & 75 & 59 \\
\hline \multicolumn{3}{|c|}{ Complication (\%) } \\
\hline slCH (PH-2 only) & 5.1 & 6.0 \\
\hline Hemicraniectomy (all cases) & 3.3 & 6.0 \\
\hline \multicolumn{3}{|c|}{$\begin{array}{l}\text { a } p<0.005 \\
\text { b } p=0.046 \\
\text { c } p=0.01 \text {, extracranial ICA includes additional cases with tandem intracranial occlusions } \\
\text { d } p<0.0001 \\
\text { Abbreviations: NIHSS: National Institute of Health Stroke Scale; ASPECTS: Alberta Stroke Program Early Computed Tomgragphic Score; IQR: Interquartile Range; ICA: } \\
\text { Internal Carotid Artery; A1: First Division of the Anterior Cerebral Artery; A2: Second Division of the Anterior Cerebral Artery; M1: First Division of the Middle Cerebral Artery; } \\
\text { M2: Second Division of the Middle Cerebral Artery; TICI: Thrombolysis in Cerebral Infarction; sICH: Symptomatic Intracerebral Hemorrhage; Ph-2: Type } 2 \text { Parenchymal } \\
\text { Hematoma. }\end{array}$} \\
\hline
\end{tabular}

Table 1: Baseline demographics, neuroimaging characteristics, and clinical course of patients undergoing EVT for anterior circulation ischemic stroke between January 2012 and December 2016, compared to the pragmatic EVT trial MR CLEAN.

$6 \% \mathrm{p}=0.55)$ and use of general anesthesia ( $44 \%$ vs. $38 \%, \mathrm{p}=0.055) \mathrm{did}$ not differ between the two groups.

\section{Patient Selection: Clinical Practice Versus Clinical Trial}

MR CLEAN enrolled patients presenting with acute ischemic stroke and CTA-proven large vessel occlusion if groin puncture could be achieved within 6 hours of symptom onset. Excluded were patients with CT evidence of intracranial hemorrhage, and the use of advanced penumbral imaging as well as ASPECTS score for patient selection were not performed. In our combined series $88 / 236$ (37\%) patients would have been excluded from this trial. 2/236 patients would have been excluded due to an NIHSS score $<2$ on presentation, $1 / 236$ patient excluded for a premorbid $\mathrm{mRS}$ of 5 (muscular dystrophy), and 1/236 patient excluded for receiving intraarterial thrombolysis with an elevated INR. Notably, all of the remaining $84 / 236$ patients would have been excluded due to a time from symptom onset to groin puncture greater than 6 hours.
Within the greater than six hour extended time window subgroup $(\mathrm{n}=84)$, we did not observe a difference in favorable outcome (mRS $0-2)$ compared to the intervention arm of MR CLEAN (28\% vs. $33 \%$, $\mathrm{p}=0.36$ ). $80 / 84$ patients in the greater than six-hour subgroup had CT perfusion completed. There was a significant difference in favorable outcome between our clinical practice and the intervention arm of the recently presented data of the extended time window embolectomy trial DAWN6, $(29.7 \%$ vs. $48.6 \%, \mathrm{p}<0.0001) .35 / 84(42 \%)$ of patients in the greater than six-hour window presented with an unknown time of onset (i.e., wake up stroke or last seen well).

\section{Discussion}

Overall, we observed similarity between the pragmatic EVT trial MR CLEAN and our clinical experience of endovascular treatment of anterior circulation large vessel occlusion at two U.S. academic comprehensive stroke centers, as measured by functional outcomes, 
recanalization rates, and complication rates. However, we also found several differences between our practices and the MR CLEAN cohort. The clinical practice group had statistically higher rates of atrial fibrillation, diabetes, and history of stroke, all of which were typically associated with worse prognosis. Furthermore, median time from symptom onset to groin puncture was longer and use of IV t-pa was lower for the combined clinical experience. Clinical outcomes were similar to MR CLEAN despite a higher proportion of patients with these conventional vascular risk factors. Notably, $28 \%$ of the patients in our subgroup excluded from MR CLEAN due to a time from symptom onset to groin puncture greater than 6 hours experienced favorable outcomes. This is similar to the proportion of favorable outcomes achieved in the intervention arm of MR CLEAN. However, the rate of dichotomized favorable outcome in this subgroup of our patient series was significantly lower than what was reported in the recent DAWN trial.6 Although 80/84 patients in our extended window subgroup received CT perfusion, RAPID software was not available to compare core infarct size with the DAWN interventional arm and treatment decisions in our practice were individualized. An additional caveat is that only $42 \%$ of our extended window embolectomy subgroup presented with unknown time of onset (wake up stroke or unwitnessed as opposed to known onset of more than six hours) in comparison to $90 \%$ of the treatment arm of DAWN. This important difference in baseline characteristics may in some part explain our worse outcomes in the extended window. Nevertheless, our findings support the use of core infarct imaging for optimal patient selection in this setting. The rate of decompressive hemicraniectomy was similar to MR CLEAN despite longer times from symptom onset to groin puncture. Rates of symptomatic intracerebral hemorrhage were also similar between the combined practice and MR CLEAN. Ultimately, our results support the external validity of MR CLEAN.

\section{Limitations}

This study has several limitations. Contemporary outcomes may be better as the clinical practice group included patients treated prior to current rapid triage protocols. Some patients were treated prior to the advent of stent-retriever technology. Although recanalization was abstracted from procedure reports and confirmed by image review, they were not scored by a core lab. We did not collect information on pre-stroke disability. CT perfusion was used for all patient selection at one center, only in extended time windows at the other center, and RAPID software was unavailable, making further quantification of the relationship between infarct core and clinical outcome difficult. A recent analysis of CT perfusion in MR CLEAN indicates that within the sixhour window the intervention arm benefited irrespective of whether a perfusion mismatch was present [13]. While a low CT ASPECTS was not an exclusion criterion in MR CLEAN, we did not treat any patients with ASPECTS of $\leq 6$ with EVT, and therefore cannot comment on this subpopulation.

\section{Conclusion}

This two-center retrospective comparison of the clinical practice of EVT for anterior circulation large vessel occlusion supports the external validity of the pragmatic endovascular trial MR CLEAN given overall similarity in clinical presentation, functional outcomes, and complications rates. Yet, in our practices we often treated patients beyond the first 6 hours from symptom onset. This suggests that the results from MR CLEAN can be replicated in practice with a longer therapeutic window for intervention.

\section{References}

1. Rothwell PM (2005) External validity of randomised controlled trials: To whom do the results of this trial apply?. Lancet 365: 82-93.

2. Campbell BC, Mitchell PJ, Kleinig TJ, Dewey HM, Churilov L, et al. (2015) Endovascular therapy for ischemic stroke with perfusion-imaging selection. $\mathrm{N}$ Engl J Med 372:1009-1018.

3. Saver JL, Goyal M, Bonafe A, Diener HC, Levy E, et al. (2015) Stent-retriever thrombectomy after intravenous t-PA vs. t-PA alone in stroke. $\mathrm{N}$ Engl $\mathrm{J}$ Med 372: 2285-2295.

4. Goyal M, Demchuk AM, Menon BK, Esa M, Rempel JL, et al. (2015) Randomized assessment of rapid endovascular treatment of ischemic stroke. N Engl J Med 372: 1019-1030.

5. Berkhemer OA, Fransen PS, Beumer D, Van den Berg LA, Lingsma HF, et al (2015) A randomized trial of intraarterial treatment for acute ischemic stroke. $\mathrm{N}$ Engl J Med 372: 11-20.

6. Jovin TG NR (2017) DAWN in full daylight. European Stroke Organisation Conference; Prague, Czech Republic.

7. Barber PA, Demchuk AM, Zhang J, Buchan AM (2000) Validity and reliability of a quantitative computed tomography score in predicting outcome of hyperacute stroke before thrombolytic therapy. ASPECTS Study Group. Alberta Stroke Programme Early CT Score. Lancet 355: 1670-1674.

8. Pexman JH, Barber PA, Hill MD, Babb JS, Siller KA, et al. (2001) Use of the Alberta Stroke Program Early CT Score (ASPECTS) for assessing CT scans in patients with acute stroke. AJNR Am J Neuroradiol 22: 1534-1542.

9. Zaidat OO, Yoo AJ, Khatri P, Tomsick TA, Von Kummer R, et al. (2013) Recommendations on angiographic revascularization grading standards for acute ischemic stroke: a consensus statement. Stroke 44: 2650-2663.

10. Wahlgren N, Ahmed N, Davalos A, Ford GA, Grond M, et al. (2007) Thrombolysis with alteplase for acute ischaemic stroke in the safe implementation of thrombolysis in Stroke-Monitoring Study (SITS-MOST): An observational study. Lancet 369: 275-282.

11. Wilson JT, Hareendran A, Grant M, Baird T, Schulz UGR, et al. (2002) Improving the assessment of outcomes in stroke: use of a structured interview to assign grades on the modified Rankin Scale. Stroke 33: 2243-2246.

12. Wilson JT, Hareendran A, Hendry A, Potter J, Bone I, et al. (2005) Reliability of the modified Rankin Scale across multiple raters: benefits of a structured interview. Stroke 36: 777-781.

13. Borst J (2016) CT Imaging in acute ischemic stroke. 